\title{
MAPPING LARGE-SCALE STRUCTURE WITH RADIO SOURCES
}

\author{
J.V. WALL \\ Royal Greenwich Observatory \\ Madingley Road, Cambridge, U.K. CB3 OEZ \\ C.R. BENN \\ Isaac Newton Group, Royal Greenwich Observatory \\ Apartado 321, 38780 Santa Cruz de La Palma, Spain \\ AND \\ A.J. LOAN \\ Institute of Astronomy \\ Madingley Road, Cambridge, U.K. CBЗ OHA
}

\section{Introduction}

In regions away from the Galactic plane, formal tests indicate an isotropic, random and independent distribution of radio sources on the sky (e.g. [1], although there are strong indications of large-scale anomalies (e.g. [2], [3]). An accommodation of these results was suggested by Shaver and Pierre [4] and by Shaver [5] who showed that the large-scale deviations could be due to the supergalaxy, a possibility which had been noted by Pauliny-Toth et al. in 1978 [6]. As to the influence of other superclusters, or indeed the cellular structure of the universe in which galaxies cluster on scales up to at least $100 h^{-1} \mathrm{Mpc}$ (e.g. [7]), at what flux-density level does this large-scale structure become apparent? Conversely, what can be learnt about structure on the largest scales through the sky distribution of radio sources? Here we describe three investigations in various stages of completion which consider these issues.

\section{A Toy Voronoi Universe}

To determine what kind of radio survey (area, flux-density level) we need in order to see the imprint of the skeleton of the universe, we modelled the large-scale structure as a three-dimensional Voronoi tessellation [8]. Poisson

M. Kafatos and Y. Kondo (eds.), Examining the Big Bang and Diffuse Background Radiations, 481-485.

(c) 1996 IAU. Printed in the Netherlands. 
Voronoi tessellations are constructed by dividing space into cells around randomly-distributed seeds, such that every point in a given cell is nearer to its seed than to any other seed. The tessellations are thus characterised by one parameter: the mean space density of seeds $\rho$. We allowed this parameter the range $50 h^{-1} \mathrm{Mpc}<\rho^{-1 / 3}<800 h^{-1}$ Mpc. Pencil-beam surveys slicing through typical Voronoi tessellations are shown in Fig. 1.
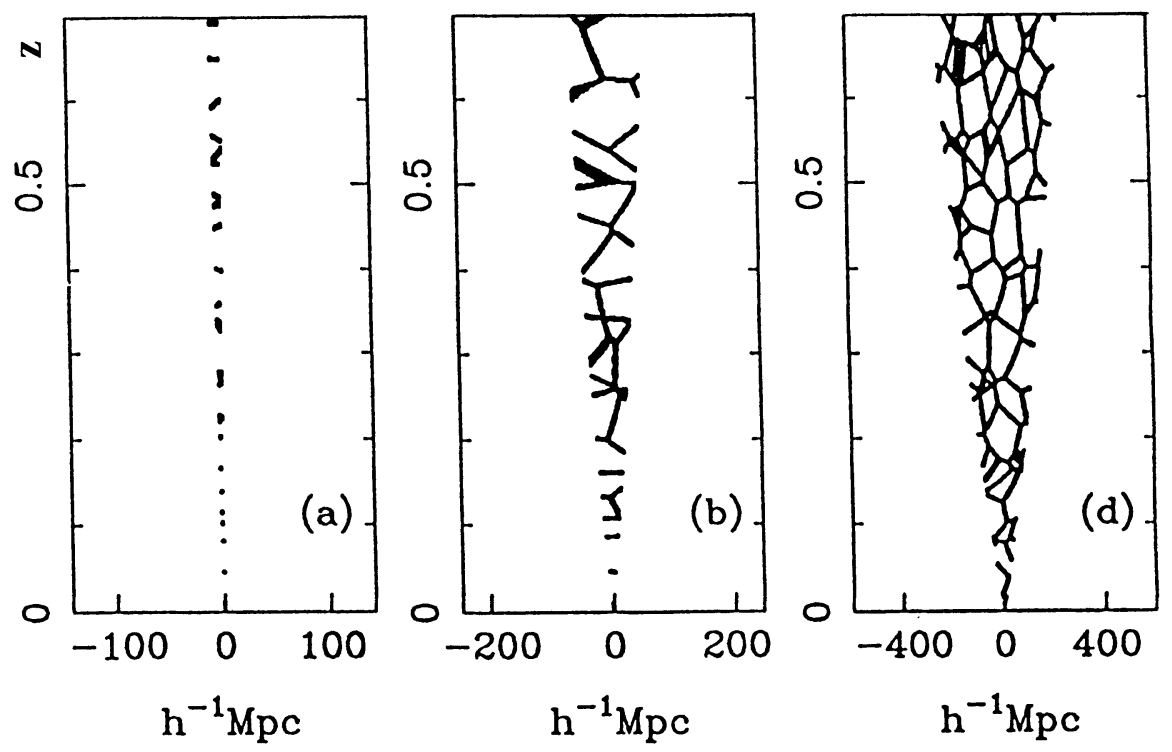

Fig. 1 Slices through Voronoi-foam simulations with $\rho^{-1 / 3}=100 h^{-1} \mathrm{Mpc}$, for pencil-beam surveys (a) $0.5^{\circ}$ in diameter, (b) $5^{\circ}$ in diameter, (c) $20^{\circ}$ in diameter. The horizontal axis is comoving $h^{-1} \mathrm{Mpc}$.

We found the predictions of the model to depend only weakly on the other main parameters: the redshift-dependent luminosity function of the radio sources to be sprinkled on the cell walls; the density parameter $\Omega$; and the distribution of radio sources within the cells.

We tested the isotropy of the sky distribution for each value of $\rho^{-1 / 3}$, at each of a range of flux densities between $10 \mu \mathrm{Jy}$ and $10 \mathrm{Jy}$, comparing the area-to-area fluctuations in counts with those expected from statistical noise for a range of survey opening angles. The results, reported in detail elsewhere [9], are as follows:

(1) The observed isotropy of the radio-source distribution at Jy, mJy and $\mu \mathrm{Jy}$ levels implies $\rho^{-1 / 3}<150 h^{-1} \mathrm{Mpc}$, with the strongest constraints currently being provided by mJy (e.g. $5 \mathrm{C}$ ) and $\mu \mathrm{Jy}$ surveys. This limit in the context of other constraints on large-scale structure occupies a critical range between those provided by galaxy surveys and by the COBE results. (2) If $\rho>\left(100 h^{-1} \mathrm{Mpc}\right)^{-3}$, area-to-area fluctuations are expected to be 
significant ( $>$ statistical noise) for e.g. $5^{\circ}$-diameter surveys at flux densities $S_{408 M H z}<10 \mathrm{mJy}$, or $0.5^{\circ}$-diameter surveys at flux densities $S_{408 M H z}<$ $0.3 \mathrm{mJy}$. These are the flux-density limits of the deepest pencil-beam radio surveys yet carried out. If the largest structures are indeed $\sim 100 \mathrm{~h}^{-1} \mathrm{Mpc}$ as optical surveys suggest, we must now be on the threshold of detecting the imprint of large-scale structure in the low-frequency radio sky.

\section{Direct sensing of cellular structure from redshift surveys of radio sources}

Radio surveys select sources over a wide range of redshifts and can thus sample the superclustering structure over very large volumes. For bright surveys, the sampling is very sparse ( $<<1$ source per supercluster), but surveys which detect sources with space density $>\sim\left(100 h^{-1} \mathrm{Mpc}\right)^{-3}$ (corresponding to $P_{408 M H z}<10^{25.5} \mathrm{WHz}^{-1}$ ) can be used to trace individual structures. What are the ideal parameters of such a radio/optical survey?

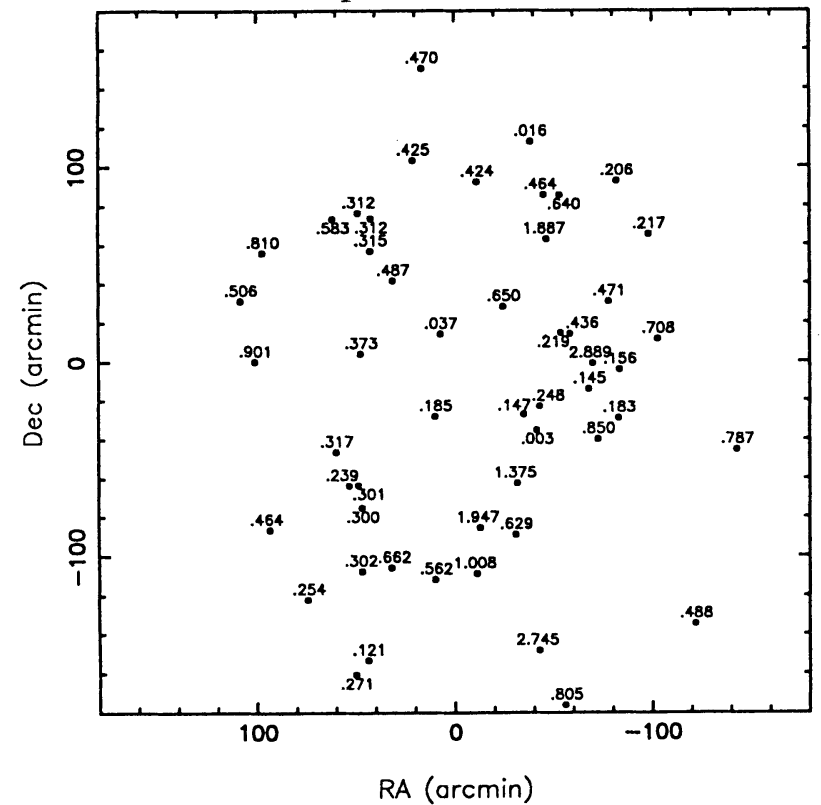

Fig. 2 Distribution on the sky of $5 \mathrm{C} 12$ sources with measured redshifts, overlapping points slightly displaced for clarity.

It is relatively simple to obtain redshifts of radio galaxies out to $z \sim$ 0.5 . The radio survey should be $>\sim$ one cell in diameter at this redshift, in order that large fractions of individual cells can be traced. This suggests a survey opening angle $\theta$ of a few degrees (given that 100 comoving $h^{-1} \mathrm{Mpc}$ at $z=0.5$ corresponds to $\theta=5^{\circ}$ ). The radio survey should also be deep enough to detect sources at this redshift with $P_{408 M H z}<<10^{25.5} \mathrm{WHz}^{-1}$, 
i.e. $S_{408 M H z}<<100 \mathrm{mJy}$.

The $5 \mathrm{C} 12$ radio survey of a $4^{\circ}$-diameter patch of sky reaches $S_{408 M H z}$ $=10 \mathrm{mJy}$; the catalogue contains $\approx 600$ sources. Redshifts have now been measured (mainly with the WHT faint-object spectrograph) for about 60 of the sources, and Fig. 2 shows their distribution on the sky. Several groups and pairs are visible. The group at $z \approx 0.315$ has projected comoving diameter $25 h^{-1} \mathrm{Mpc}$, while others have projected diameters ranging 10 $70 h^{-1} \mathrm{Mpc}$.

\section{Large-area surveys: two-point correlation}

The third investigation is two-point correlation-function analysis, particularly well-suited to surveys of irregular areas, such as all-sky surveys after excision of regions of low Galactic latitude and confused regions near bright/extended sources. Two surveys at $5 \mathrm{GHz}$, each covering $\approx$ one hemisphere (e.g. [10],[11]), now catalogue $\approx 10^{5}$ sources. New correlation results (Fig. 3) confirm the apparent signal previously noted in this type of analysis from the northern region alone [12][13].

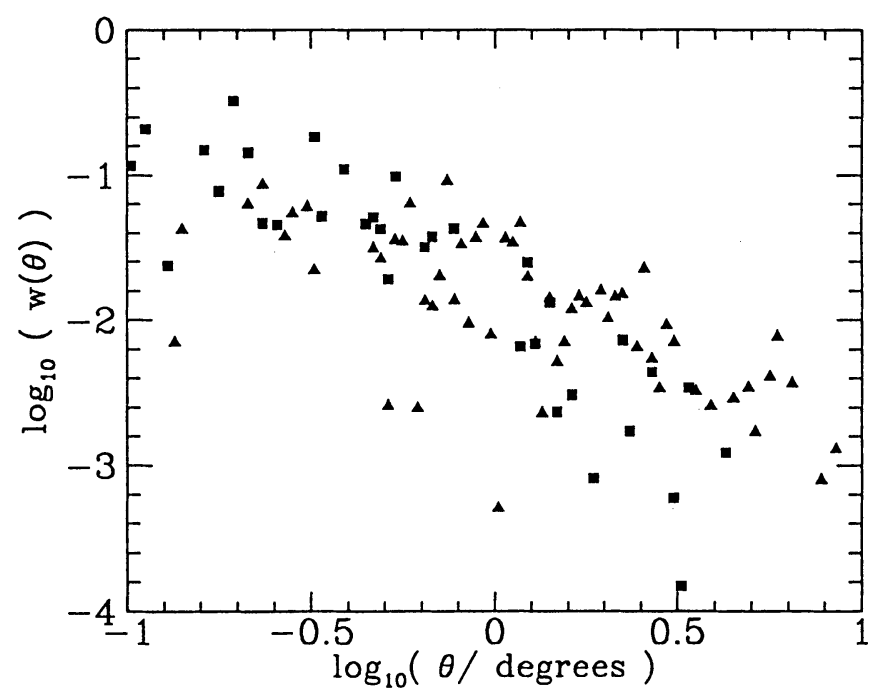

Fig. 3 Preliminary two-point correlation functions for sources of flux density $>50 \mathrm{mJy}$ in different areas of the Greenbank - PMN 5-GHz survey.

Further analysis is needed to distinguish intrinsic non-randomness from instrumental and geometrical effects [14]. If intrinsic non-randomness is confirmed, Limber's equation (e.g. [15]) can be applied to derive the power spectrum of the inherent clustering, because the nature and redshift distribution of the $5-\mathrm{GHz}$ sources are reasonably well established [12][16]. 


\section{References}

1. Webster, A.S., 1977, in Radio Astronomy and Cosmology, Proc IAU Symp. 74, ed. Jauncey, D.L., Reidel, 75.

2. Wall, J.V., 1977, in Radio Astronomy and Cosmology, Proc IAU Symp. 74, ed. Jauncey, D.L., Reidel, 55.

3. Pauliny-Toth, I.I.K., 1977, in Radio Astronomy and Cosmology, Proc IAU Symp. 74, ed. Jauncey, D.L., Reidel, 63.

4. Shaver, P.A. and Pierre, M., 1989, Astron. Astrophys., 220, 35.

5. Shaver, P.A., 1991, Austr. J. Phys., 44, 759.

6. Pauliny-Toth, I.I.K., Witzel, A., Preuss, E., Kühr, H., Kellermann, K.I., Fomalont, E.B. and Davis, M.M., 1978, Astron. J., 83, 451.

7. Giovanelli R. and Haynes M.P., 1991, Ann. Rev. Astron. Astrophys., 29, 499.

8. Icke V. and van de Weygaert R., 1991, Q. J. R. astr. Soc., 32, 85.

9. Benn C.R. and Wall J.V., 1994, Mon. Not. R. astr. Soc., in press.

10. Gregory, P.C. and Condon, J.J., 1991, Astrophys. J. Suppl. Ser., 75, 1011.

11. Griffith, M.R. and Wright, A.E., 1993, Astron. J., 105, 1666.

12. Wall, J.V., Rixon, G.T. and Benn, C.R., 1993, in Observational Cosmology, eds Chincarini, G. et al., ASP Conf. Ser. 51, 576.

13. Kooiman, B.L., Burns, J.O. and Klypin, A.A., 1994, Astrophys. J., in press.

14. Loan, A.J., Wall, J.V. and Lahav, O., in preparation.

15. Fall, S.M., 1979, Rev. Mod. Phys., 51, 21.

16. Dunlop, J.R. and Peacock, J.A., 1990, Mon. Not. R. astr. Soc., 247, 19. 